\title{
Managing Hotspot Regions in Wireless/Cellular Networks with Partial Coverage Picocells
}

\author{
Jingxiang Luo and Carey Williamson \\ Department of Computer Science, University of Calgary \\ Calgary, AB, Canada \\ jingx.luo@gmail.com, carey@cpsc.ucalgary.ca
}

\begin{abstract}
In typical deployments of wireless and cellular networks, traffic hotspots can arise from the non-uniform geographic distribution of the mobile users, and the heavy-tailed nature of their network usage patterns. These hotspots of activity can degrade system performance, by increasing network utilization, wireless interference, call blocking, and even call dropping from failed handoffs for mobile users. One approach for network providers is to deploy a picocell as a targeted solution for a specific geographic region of interest. In this paper, we develop an analytical model to characterize the performance of a wireless/cellular network augmented with picocells. Our numerical results illustrate several tradeoffs between network cost, call blocking, and call dropping. Our model provides insights on how many picocells to deploy, where to place them, and their performance impacts.
\end{abstract}

\section{Categories and Subject Descriptors}

C.2.1 [Computer-Communications Networks]: Network Architecture and Design; C.2.2 [Computer-Communications Networks]: Network Protocols; C.4 [Performance of Systems]: Performance Attributes

\section{General Terms}

Design, Management, Performance

\section{Keywords}

Wireless Networks, Cellular Networks, Picocells

\section{INTRODUCTION}

The past two decades have witnessed a rapid development of cellular networks. As cellular systems are gaining popularity, the traffic demand has increased significantly while the available wireless bandwidth is still scarce. Many methods $[5,11,12,15,17]$ have been proposed to promote the efficient use of wireless bandwidth while maintaining Quality of Service (QoS) standards and low operating costs.

Permission to make digital or hard copies of all or part of this work for personal or classroom use is granted without fee provided that copies are not made or distributed for profit or commercial advantage and that copies bear this notice and the full citation on the first page. To copy otherwise, to republish, to post on servers or to redistribute to lists, requires prior specific permission and/or a fee.

MobiWac'08, October 30-31, 2008, Vancouver, BC, Canada.

Copyright 2008 ACM 978-1-60558-055-5/08/10 ...\$5.00.
In a wireless cellular network, call activity can be more intensive in some areas than others. These high-traffic areas are called hotspot regions. To address this issue, we use two sets of base stations (BS): one set consists of Essential Base Stations (EBS) that cover the whole area, as in traditional cellular networks, while the other set consists of Complementary Base Stations (CBS) that cover the hotspot region.

In the literature, there are several pioneering works related to this approach, which is typically described as a two-layer hierarchical cellular network $[8,11]$. To align with the terminology used in [8], the large circular coverage area of an EBS at the parent level is referred to as a macrocell, while the small coverage area of a CBS at the child level is called a picocell. The main difference in our model is that only part of the macrocell area, namely the hotspot region, is covered with picocells.

Mobile users initiate calls in the system. Following the terminology in [8], a call in its first attempt to establish a connection with a BS is referred to as a new call. A call can be redirected as follows. A call attempting to connect to one set of BS is allowed to be redirected to the other set of BS as an overflow call. An overflow call may later revert back to the original BS as a takeback call. Handoff calls are requests to transfer calls between two CBS at the same level or between two EBS at the same level.

We consider traffic management issues between mobile users and base stations. Considering typical voice traffic on either an uplink or a downlink, we assume that a link is divided into a fixed number of channels, and each incoming call occupies one channel. We assume that traffic is not evenly distributed geographically; rather, there is a hotspot region where the traffic is the most intense. We establish a set of CBS that collectively cover the hotspot area. Outside this area only the coverage of EBS is available. This is referred to as the $E B S-C B S$ model. We use the term partial coverage picocells to emphasize that the CBS covers only part of the area within the macrocell.

Partial CBS coverage raises a problem not seen in classical hierarchical cellular networks. Consider a mobile user that moves from a CBS-covered area to an area without CBS coverage. Obviously, such a user cannot succeed in requesting a handoff to another CBS, since there is none. Handing over to the EBS is the only possibility. We will illustrate that it is possible to achieve satisfactory performance under our proposed scheme, and explore the tradeoffs between system performance improvement and the system cost.

No operational research on traffic management schemes can be complete without proper methodologies for evaluat- 
ing cellular network performance. Prior works have been done on general methodologies [1,9], as well as building models for particular flows, such as handoff calls [2] and overflow calls [10]. The latter two works devise ways of capturing the correlations between different calls, or the correlation between incoming calls and the number of ongoing calls. Since these studies have shown only limited effects of correlations on system performance metrics, we follow the simple approach for performance assessment in this study [9].

The rest of this paper is organized as follows. Section 2 provides a brief synopsis of prior related work. Section 3 describes our EBS-CBS model, its model parameters, and traffic characteristics. Section 4 carries out traffic analysis through a series of balance equations, and develops an iterative algorithm to evaluate system performance. Section 5 presents numerical results. Section 6 concludes the paper.

\section{RELATED WORK}

Several authors have studied mechanisms to enhance system performance in cellular networks. In [7], the advantage of priority handoff calls was established through detailed performance comparison with non-prioritized handoffs. Handoffs in the context of two-layer hierarchical networks was discussed in [15]. Early work [8] allows unidirectional movement of redirected calls (i.e., calls overflowing from picocells to macrocells), while later work [12] allows overflows in both directions. Takeback strategies for overflow calls reverting to their original BS are introduced later [14]. Good reference works appear in [8, 11], which include policies like handoff priority access, overflow limitation, and reversible capability.

Other researchers have explored strategies for choosing the initial BS for a mobile user. In [11], the authors advocated speed-sensitive choice, which assigns slow-moving mobile users to picocells and fast-moving ones to macrocells. In [5], a simple strategy is used to direct all calls by default to picocells; the macrocells are used for overflow calls only. Many later proposals are variants of these two approaches.

In most of these recent studies, the intent of picocells is to increase system capacity. To mitigate the side-effect of more frequent handoffs among picocells, different strategies have been introduced.

Geographically homogeneous traffic is the standard assumption in these studies. Unfortunately, this convenient assumption does not pay sufficient attention to the measured patterns of traffic and mobility [6, 16] (e.g., mobile users and active calls concentrated in hotspot areas).

From the perspective of performance, hotspot regions deserve attention. A hotspot typically degrades overall network performance, due to the high load imposed in areas with intensive call traffic. These issues are carefully addressed in our paper.

\section{NETWORK MODEL}

This section describes the EBS-CBS model and specifies its associated parameters.

\subsection{Overview}

Figure 1 provides a conceptual illustration of our cellular network system model. In particular, it illustrates the concept of a hotspot region, as well as the different situations that can arise for mobile users competing for finite wire- less resources. An Essential Base Station (EBS) provides traditional wireless/cellular network coverage to a large circular region called a macrocell. Any mobile user within this macrocell can be served by the EBS. However, the traffic demand on the EBS may be non-uniform within the macrocell. In particular, the shaded area in Figure 1 depicts a hotspot region: an irregularly-shaped geographic area within the macrocell that generates higher traffic load than other areas of the macrocell.

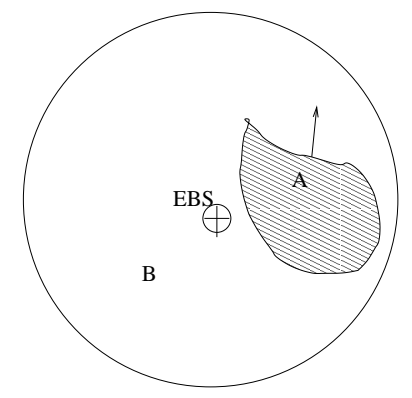

Figure 1: A diagram of partial CBS coverage

Because of the geographic clustering of the users in the hotspot area, servicing these users from the EBS may require significant transmission power, which produces higher interference for other users in the macrocell, perhaps compromising their QoS. The hotspot region is a candidate location for a picocell, wherein a low-cost (perhaps even portable) Complementary Base Station (CBS) is deployed to service users in that area. If economically viable, more than one CBS could be deployed to service the hotspot region. This approach can offload the EBS from servicing those users, and improve the QoS for all users in the macrocell.

Partial CBS coverage is a key concept in our model, which raises new issues in traffic management. CBS coverage is guaranteed only in the hotspot area. Because of partial CBS coverage, a mobile user A that resides in the hotspot area may encounter different situations than another mobile user B residing outside the hotspot area. For example, if A connects to a CBS but later moves out of the hotspot area, then transferring the call to the EBS may be necessary (since there is no CBS coverage outside the hotspot region). Thus the EBS provides additional resources that are available to user A, if needed. In contrast, a mobile user B that experiences a rejected call at the EBS cannot overflow to a CBS, unless it resides in the hotspot region.

We focus on the management of traffic redirection and priority issues, especially on those in connection with partial CBS coverage. For this purpose, it is of primary interest to quantify the area of hotspot regions in a macrocell, and how many CBS are needed to cover hotspot regions. We assume that each picocell is completely contained in a macrocell. Realistically, a picocell may overlap with other macrocells, leading to slightly lower coverage efficiency and more wireless interference. These detailed issues are beyond the scope of this initial study.

\subsection{Assumptions}

In our model, we make the following basic assumptions:

- Each EBS covers a large circular area called a macrocell. Each CBS covers a (relatively) small area called a 
picocell. Each picocell is completely contained within a macrocell.

- Call arrivals follow a Poisson process. Each call in the system requires one unit of channel capacity, either at the EBS or the CBS.

- Traffic heterogeneity exists between the hotspot area and the rest of the macrocell. Within the hotspot region, all traffic is evenly distributed.

- There are two types of mobile users. Type-1 (general) users can appear anywhere within the macrocell, and are uniformly distributed. Type- 2 users have strong affinity for the hotspot area; they appear in the hotspot area with probability $\phi$, and elsewhere in the macrocell with probability $1-\phi$. Regardless of the region in which they appear, their presence is uniformly distributed over the region.

- Calls initiated by Type-1 users are first directed to an EBS, regardless of their location. Calls initiated by Type-2 users are first directed to a CBS if the user is in the hotspot area, and to the EBS otherwise.

- Call requests that are rejected by a CBS due to insufficient resources are allowed to overflow to the EBS. Similarly, call requests rejected by an EBS can overflow to a CBS, if appropriate. Overflow calls can be reclaimed by the rightful $\mathrm{BS}$ when resources permit; this process is called a takeback.

- Handoff calls and takeback calls are given higher priority than new calls, while overflow calls are given lower priority than new calls.

\subsection{System Operation}

We consider one representative EBS among the many in a typical cellular system. This EBS has $K \geq 1$ picocells, each with its own CBS. We normalize the coverage area of the macrocell to 1 unit, and consider the size of the hotspot region relative to that of the macrocell.

Suppose $K$ identically-sized picocells, each with area $\epsilon$, collectively cover a hotspot region with area $A<1$. Let

$$
\alpha=\frac{A}{K \epsilon}
$$

denote the coverage efficiency. Due to overlaps between picocell coverage areas, we typically have $\alpha<1$.

The operational rules for admitting and redirecting call requests in the EBS-CBS system are discussed next.

\subsubsection{Macrocell Operation (EBS)}

There are a total of $C_{m}$ channels in the EBS. A new call to the EBS is rejected if no channel is available, or if the number of ongoing calls exceeds a threshold $C_{n}$. A rejected call overflows to the closest CBS if the mobile user initiating the call currently resides in the hotspot region; otherwise, the call is removed from the system. Hence this scheme is called partial overflow. The same principle applies to any handoff calls received from another EBS.

An overflow call from a CBS is rejected by the EBS if no channel is available, or if the number of ongoing overflow calls exceeds a threshold $C_{o}$. A takeback call from a CBS is rejected and continues to exploit a channel at the CBS when no channel is available at the EBS.
A mobile user moving out of the coverage area of an EBS requests a transfer of the call to a neighbouring EBS.

\subsubsection{Picocell Operation (CBS)}

There are a total of $C_{m}^{\prime}$ channels in each CBS. A new call is rejected when no channel is available or when there are more than $C_{n}^{\prime}$ ongoing calls. A rejected new call attempts to overflow to the EBS. A handoff call from another CBS is rejected only if no channel is available at this CBS; in such a case, it attempts to overflow to the EBS. An overflow call from the EBS is rejected by a CBS if no channel is available, or if there are more than $C_{o}^{\prime}$ ongoing calls. When no channel is available in the CBS, a takeback call from an EBS fails and the call continues to use an EBS channel.

The partial CBS coverage has the following consequence. When a mobile user moves out of a picocell, the user may enter another picocell within the hotspot region, or may leave the hotspot region entirely (vacating the CBS coverage area). For the latter case, if the mobile user has an ongoing call, it will request a transfer to the EBS as a privileged overflow.

The reason for privileged overflows is that no other obvious alternative works well. One possible choice is to overflow to the EBS. Unfortunately, overflows are typically assigned low priority in traffic management, which implies a high failure rate (i.e., excessive dropping) for overflows. Another alternative is to mark calls redirected to EBS in this situation as handoffs. Although avoiding the disadvantage just mentioned, a new problem arises, since redirected calls may remain arbitrarily long while consuming EBS channels (unless they are distinguished somehow from calls originated at the EBS). Consequently, this leads to higher load at the EBS, and lower utilization of CBS channels.

If no channel is available in an EBS, or the total number of ongoing calls exceeds a designated threshold $C_{p}$, then any privileged overflow call is rejected and removed from the system.

In the system configuration, we set:

$$
\begin{aligned}
& C_{o}<C_{n}<C_{p}<C_{m} \\
& C_{o}^{\prime}<C_{n}^{\prime}<C_{m}^{\prime}
\end{aligned}
$$

to ensure that priorities between new calls and redirected calls are respected.

Note that takebacks occur at picocell boundaries. Whenever a mobile user crosses a picocell boundary, the system attempts to revert overflow calls back to the original BS.

\subsubsection{Picocell Placement}

Picocell placement is a network optimization problem that seeks the best possible system performance with as few CBS as possible. We study how the system performance changes in response to changing $K$ and $\epsilon$. From the planning perspective, we seek a configuration that meets the performance requirements with minimal additional cost.

Our model accounts for traffic heterogeneity, partial CBS coverage, and their implications. We develop the model within one macrocell, hence we do not address issues arising between macrocells, such as the potential overlap between two EBS, and picocells bordering several EBS. The overlap between two EBS has been studied in $[8,17]$, under the assumption of homogeneous traffic. It is of practical importance to study models that incorporate both multiple macrocells and traffic heterogeneity for optimization of sys- 
tem performance and/or cost. This topic deserves further attention in the future.

\subsection{Model Parameters and Notation}

In this section, we present the parameters used to define the EBS-CBS system model and its traffic characteristics. The purpose of the model is to develop and evaluate a highlevel traffic management scheme, while deferring practical implementation issues (e.g., user location tracking) to future work. A summary of our model notation appears in Table 1 and Table 2.

Let $\lambda_{n}$ denote the aggregate arrival rate of new calls to the EBS. Similarly, let $\lambda_{n}^{\prime}$ denote the aggregate arrival rate of new calls to the $K \mathrm{CBS}$ in the hotspot region. We assume that $\lambda_{n}^{\prime}>\lambda_{n}$.

The cell residence time is the duration that a mobile user stays within a cellular coverage area before moving elsewhere. Obviously, the smaller a picocell is, the shorter is the expected time that a mobile user spends within it. However, this relationship is not necessarily linear. Let $\bar{R}$ represent the mean time that a mobile user resides in a macrocell, and $\bar{R}^{\prime}$ represent the mean time in a picocell. We assume that:

$$
\frac{\bar{R}^{\prime}}{\bar{R}}=\epsilon^{\beta}=\left(\frac{A}{K \alpha}\right)^{\beta} \quad(0 \leq \beta \leq 1) .
$$

where $\beta$ is a mobility parameter, and the second equality follows via substituting Equation 1 into the first equality.

If the mobility of a user is completely random, then the cell residence time is proportional to the size (area) of the cell $(\beta=1.0)$. However, if a mobile user moves with a linear trajectory, then the cell residence time is proportional to the diameter of a cell $(\beta=0.5)$. A realistic situation may fall between these two extremes. Hence we allow $0.5 \leq \beta \leq 1$.

The next two model parameters relate to partial CBS coverage and its consequences. Among all unsuccessful call requests to an EBS, define $\tau$ as the fraction that attempt overflow to a CBS (the rest are immediately removed). If mobile users are equally likely in any location, then $\tau \approx A$. From our assumption, mobile users are more likely to appear in the hotspot region. Hence we can assume:

$$
\tau \geq A \text {. }
$$

Among all handoffs from a CBS, define $\chi$ as the fraction directed to another CBS (the rest are directed to an EBS). From our assumption, mobile users are more likely in the hotspot area. If handoffs to any location in the macrocell are equally likely, then:

$$
\chi \geq \frac{A-\epsilon}{1-\epsilon},
$$

where the numerator is the area within the hotspot but outside the current picocell, and the denominator is the total area within the macrocell but outside of the current picocell. A conservative estimate of $\chi$ is achieved with equality in this relation; this happens if mobile users appear equally likely in any location. For simplicity, we also assume that the handoff behaviour out of each picocell is statistically identical.

In our model, there is a difference between system-level performance and user-perceived performance, with respect to call blocking and dropping. These differences arise since some calls have a second chance at being accommodated, even if they are initially blocked at one BS. Furthermore, some calls may be redirected more than once. A BS views each such instance as a separate call event, while a user does not; they are only concerned with the ultimate probability of completing a call or not.

To study user-perceived performance, it is necessary to identify which user initiates a call. New call arrivals to the EBS arise from Type-1 users, and from Type- 2 users who initiate calls outside the hotspot region. Let $\lambda^{u_{1}}$ be the new call rate of Type- 1 users. Let $\lambda^{u_{2}}$ be the new call rate of Type- 2 users, and let $\phi$ be the probability that Type- 2 users initiate a call from within the hotspot area. Then:

$$
\begin{aligned}
& \lambda_{n}=\lambda^{u 1}+(1-\phi) \lambda^{u 2} \\
& \lambda_{n}^{\prime}=\phi \lambda^{u 2} .
\end{aligned}
$$

\subsection{Performance Metrics}

To recap the discussion so far, we introduced five types of calls, namely: new calls, handoff calls, and three types of redirected calls, including overflow calls, privileged overflow calls, and takeback calls. Each type of call is treated as a stream of calls in our model.

The following notation is used throughout the rest of the paper. Let $\varphi$ be an enumerative type variable taking values from set $\mathcal{E}=\{n, h, o, p, t\}$, where $n$ stands for new calls, $h$ stands for handoff calls, o stands for overflow calls, $p$ stands for privileged overflows, and $t$ stands for takeback calls.

The notations used for macrocells (EBS) and for picocells (CBS) are analogous, with a / (prime) added to distinguish them. For example, $\lambda_{\varphi}$ is the call arrival rate for stream $\varphi$ at an EBS, while $\lambda_{\varphi}^{\prime}$ is the call arrival rate for stream $\varphi$ at a CBS.

For each traffic stream, we define the following metrics:

- $p_{\varphi}^{r}$ - the rejection probability of stream $\varphi$;

- $(\mathrm{CT})_{\varphi}$ - the carried traffic of stream $\varphi$ in the EBS; the total carried traffic in an EBS is $(\mathrm{CT})=\sum_{\varphi}(\mathrm{CT})_{\varphi}$.

Following the terminology in the literature, the rejection probabilities are named for each stream $\varphi$ : blocking of new calls $\left(p_{n}^{r}\right)$, dropping of handoff calls $\left(p_{h}^{r}\right)$, failed overflow probability $\left(p_{o}^{r}\right)$, and takeback failure probability $\left(p_{t}^{r}\right)$.

Users are not concerned with whether a call has been redirected, as these are just traffic management details. Metrics of interest to users are the final blocking probability of new calls as well as the final dropping probability of handoff calls. We introduce the following notation:

$$
P_{\text {block }}^{u 1}, P_{d r o p}^{u 1}, P_{\text {block }}^{u 2}, P_{d r o p}^{u 2}
$$

where the superscripts $u 1$ and $u 2$ refer to Type- 1 and Type- 2 users, respectively.

The system costs for setup and operation are also important in our model. Briefly, the following costs are introduced in our proposed scheme: (1) the cost of the EBS, which is fixed, assuming a constant number of channels per EBS; (2) the CBS set-up cost, which is proportional to $K$; and (3) the CBS operation cost, which is proportional to the number of available channels and the area of the hotspot.

\section{MODEL ANALYSIS}

This section presents the analysis of our traffic management scheme, and develops an iterative algorithm to evaluate the performance metrics. 
Table 1: Model Notation for System Configuration Parameters

\begin{tabular}{|c|l|}
\hline Symbol & Description \\
\hline \hline$C_{m}$ & Channel capacity (number of channels) for Essential Base Station (EBS) \\
$C_{n}$ & Threshold value for admitting new calls at EBS \\
$C_{o}$ & Threshold value for admitting overflow calls at EBS \\
\hline$C_{m}^{\prime}$ & Channel capacity (number of channels) for a Complementary Base Station (CBS) \\
$C_{n}^{\prime}$ & Threshold value for admitting new calls at a CBS \\
$C_{o}^{\prime}$ & Threshold value for admitting overflow calls at a CBS \\
\hline$A$ & Proportion of macrocell area occupied by traffic hotspot \\
$K$ & Number of CBS picocells deployed within a macrocell \\
$\epsilon$ & Geographic area covered by each picocell \\
$\alpha$ & Relative efficiency of hotspot coverage using picocells \\
\hline
\end{tabular}

\subsection{State Variables}

Let $N(t)$ denote the number of ongoing calls in an EBS at time $t$. We divide ongoing calls in a BS into five streams, as given by $\mathcal{E}$. We have $N(t)=i(t)+j(t)+k(t)+l(t)+l_{p}(t)$, where $i, j$, and $k$ are respectively the number of ongoing new calls, handoff calls, and takeback calls, while $l$ and $l_{p}$ are the number of overflow calls and privileged overflow calls, respectively. Associated with a CBS, there is another stochastic process denoted $N^{\prime}(t)$, which is similar to $N(t)$ except that it is observed in one picocell.

For queueing networks, an analysis based on traffic balance equations has been developed, which yields a productform solution. Our setting is slightly different, but very similar to [9], where the solution can be approximated by a product-form solution. We follow the latter approach.

First we establish the traffic characteristics in the model (e.g., arrivals to and departures from each BS). It is essential to describe the traffic and performance in both the macrocell and the picocells.

Focusing on a macrocell (an area covered by an EBS), we have the following set of traffic characteristics and performance metrics defined on $\mathcal{E}$ :

- $\lambda_{\varphi}$ - the arrival rate of stream $\varphi$ in an EBS;

- $\bar{T}_{\varphi}$ - the mean channel holding time of stream $\varphi$ in a EBS (note that a channel may be released if a mobile user moves out of the coverage area);

- $h_{\varphi}$ - the probability that a mobile user moves out of the coverage area of an EBS before an ongoing call terminates (i.e., a handoff request is necessary); and

- $v_{\varphi}$ - the probability that a mobile user moves across the boundary of a picocell while a call is in overflow state (i.e., the system initiates a takeback attempt).

Note, however, that the characteristics listed above can be synthesized from the random variables introduced below:

- $T^{0}$ - call duration (i.e., from call initiation until call termination);

- $R$-residence time in a macrocell (i.e., from the time a mobile user enters a cell until it moves out of that cell)

The characteristics above have their counterparts in a picocell. For example, $R^{\prime}$ is the residence time in a picocell, and $\bar{T}_{\varphi}^{\prime}$ is the mean channel holding time of stream $\varphi$ in a CBS.

\subsection{Channel Holding Times}

A call can change its stream type during its lifetime. By definition, $T_{\varphi}$ is the time that a call of stream $\varphi$ holds a channel of a BS. Referring to Figure 2, let us examine an example of how $T_{\varphi}$ relates to $T^{0}$ and $R$ :

1. $\varphi=n: T_{n}$ is the time from the instant of call initiation until the instant that the mobile user moves out of the current cell.

2. $\varphi=h: T_{h}$ is the time from the instant that the mobile user moves into this cell until the instant of call termination or the mobile user leaving the cell, whichever comes first.

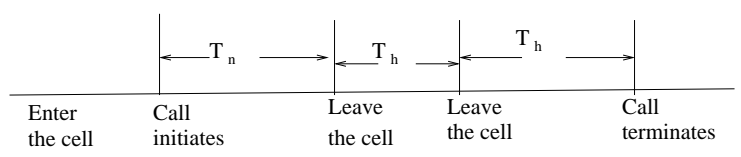

Figure 2: Diagram illustrating channel holding times

Let $\left(T^{0}\right)^{\sigma}$ be the remaining time of $T^{0}$ (i.e., the tail of $T^{0}$ from a random instant), and let $(R)^{\sigma}$ be the remaining residence time. If both $T^{0}$ and $R$ are exponentially distributed, then $\left(T^{0}\right)^{\sigma} \sim T^{0}$ and $(R)^{\sigma} \sim R$, from the memoryless property. In this case, we actually have identical distributions for $T_{\varphi}$ 's, hence we may omit the index $\varphi$. The calculation of $\bar{T}_{\varphi}$ and $h_{\varphi}$ can be simplified, as follows:

$$
\begin{aligned}
\frac{1}{\bar{T}} & =\frac{1}{\bar{T}^{0}}+\frac{1}{\bar{R}} \\
\frac{1}{h} & =1+\frac{\bar{R}}{\bar{T}^{0}}
\end{aligned}
$$

In the general case, the following relations hold:

$$
\begin{aligned}
T_{\varphi}^{0} \sim T^{0}, \quad \text { if } \varphi \text { is } \mathrm{n} \\
T_{\varphi}^{0} \sim\left(T^{0}\right)^{\sigma}, \text { otherwise } \\
R_{\varphi} \sim R, \text { if } \varphi \text { is h or t } \\
R_{\varphi} \sim(R)^{\sigma}, \text { otherwise }
\end{aligned}
$$

In deciding call holding times $\bar{T}_{\varphi}$ and handoff probabilities $h_{\varphi}$, the call durations and the cell residence times play fundamental roles. We have:

$$
\begin{aligned}
& T_{\varphi}=\min \left(T_{\varphi}^{0}, R_{\varphi}\right) \\
& h_{\varphi}=\mathbf{1}\left(R_{\varphi}<T_{\varphi}^{0}\right)
\end{aligned}
$$


Table 2: Model Notation for Traffic and Mobility Parameters

\begin{tabular}{|c|c|}
\hline Symbol & Description \\
\hline $\bar{T}^{0}$ & Random variable representing call duration \\
\hline$R$ & Random variable representing cell residence (occupancy) time \\
\hline$\sigma$ & Superscript used to refer to tail of distribution \\
\hline$(R)^{\sigma}$ & Tail of cell residence time distribution \\
\hline$\phi$ & Fraction of Type-2 (hotspot affinity) calls that initiate from within hotspot \\
\hline$\tau$ & Fraction of rejected calls at an EBS that overflow to a CBS \\
\hline$\chi$ & Fraction of handoffs to another CBS (the rest become privileged overflows to EBS) \\
\hline $\mathcal{E}$ & Set of call-stream types, $\mathcal{E}=\{\mathrm{n}, \mathrm{h}, \mathrm{o}, \mathrm{p}, \mathrm{t}\}$ \\
\hline$\varphi$ & Subscript index for call stream type: $\varphi \in \mathcal{E}$ \\
\hline$T_{\varphi}$ & Random variable denoting that a call of stream $\varphi$ holds a channel \\
\hline$\Omega$ & System state space \\
\hline$\Xi_{\varphi}$ & Rejection set (a subset of the state space in which calls of stream $\varphi$ are rejected) \\
\hline$\lambda_{\varphi}$ & Arrival rate of stream $\varphi$ to EBS \\
\hline$\Lambda_{\varphi}$ & Load imposed on EBS by stream $\varphi$ \\
\hline$p_{\varphi}^{r}$ & Rejection probability of stream $\varphi$ at EBS \\
\hline$N(t)$ & Total number of ongoing calls at time $t$ in EBS \\
\hline$\pi$ & Joint equilibrium distribution at EBS \\
\hline$(\mathrm{CT})$ & Total carried traffic by EBS per macrocell \\
\hline$\Lambda_{\varphi}^{\prime}$ & Load imposed on a CBS by stream $\varphi$ \\
\hline$\lambda_{\varphi}^{\prime}$ & Arrival rate of stream $\varphi$ to a CBS \\
\hline$p_{\varphi}^{\prime r}$ & Rejection probability of stream $\varphi$ at CBS \\
\hline$N^{\prime}(t)$ & Total number of ongoing calls at time $t$ in CBS \\
\hline$\pi^{\prime}$ & Joint equilibrium distribution at CBS \\
\hline$(\mathrm{CT})^{\prime}$ & Total carried traffic by CBS per macrocell \\
\hline$h_{\varphi}$ & Macrocell handoff probability (i.e., mobile user leaves macrocell before an ongoing call terminates) \\
\hline$h_{\varphi}^{\prime}$ & Picocell handoff probability (i.e., mobile user leaves picocell before an ongoing call terminates) \\
\hline$v_{\varphi}$ & Takeback probability (i.e., mobile user leaves picocell before an overflow call terminates) \\
\hline$u 1$ & Superscript used to refer to Type-1 (general) users \\
\hline$u 2$ & Superscript used to refer to Type- 2 (hotspot affinity) users \\
\hline
\end{tabular}

where $\mathbf{1}($.$) is the indicator function. In the above formula-$ tion, the first equation says that a call initiated by a mobile user occupies a channel until the call terminates or the mobile user moves out of the cell, whichever comes first. The second equation says that a handoff is requested whenever the mobile moves out of the cell before the call terminates.

Taking expectations for the foregoing equations gives:

$$
\begin{aligned}
\bar{T}_{\varphi} & =\mathrm{E}\left[\min \left(T_{\varphi}^{0}, R_{\varphi}\right)\right] \\
h_{\varphi} & =\operatorname{Pr}\left(R_{\varphi}<T_{\varphi}^{0}\right)
\end{aligned}
$$

For each $\varphi$, given that the distribution of $T^{0}$ and the distribution of $R$ are known, the relations for $\bar{T}_{\varphi}$ and $h_{\varphi}$ can be derived. There are several methods for doing this, including Laplace transforms and generating functions [17].

\subsection{Traffic Balance Equations}

Following the previous notation, we use the subscript $\varphi$ to denote traffic characteristics related to stream $\varphi \cdot \lambda_{\varphi}$ and $\bar{T}_{\varphi}$ are respectively the arrival rate and mean channel holding time at an EBS. $\bar{T}_{\varphi}^{\prime}$ is the mean holding time of a channel in a CBS, but note that $\lambda_{\varphi}^{\prime}$ is the arrival rate of call stream $\varphi$ to all $K$ CBS within a macrocell (consistent with Section 3.4).

Throughout the rest of the paper (unless otherwise mentioned), exponential distributions for call duration and cell residence times are assumed. With these assumptions, the $h_{\varphi}$ probabilities become identical, regardless of $\varphi$. Similar can be said for $v_{\varphi}, h_{\varphi}^{\prime}$ and $v_{\varphi}^{\prime}$. Hence, we drop all subscripts in these probabilities. (Extending to the case of non- exponential distributions is straightforward, but we have to add back the subscript $\varphi$ where appropriate.)

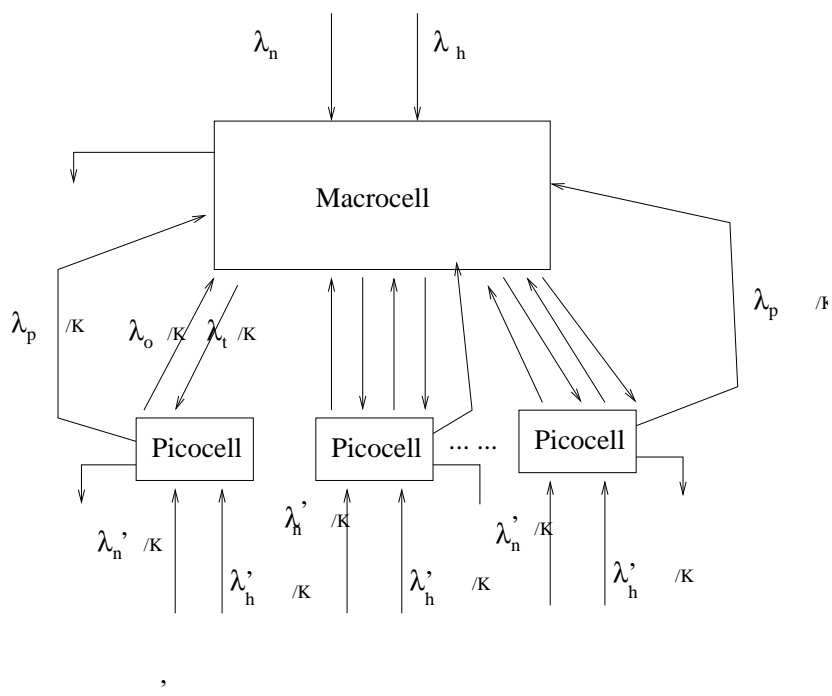

Figure 3: A block diagram of the traffic in our model (with one macrocell, $K$ picocells)

Two groups of balance equations are needed: one group for an EBS, and the other for a CBS. The block diagram in Figure 3 shows the arrivals and transfers between base 
stations, to illustrate an overall picture of the traffic in the model. In particular, please note that the fraction of partial overflow is $\tau$, and that the fraction of partial handoff is $\chi$, as discussed in Section 3.4.

\subsubsection{Macrocell Model (EBS)}

Handoffs between two EBS are always permitted, and overflows from a CBS to EBS are always allowed. However, if a call connects to a CBS, and the mobile user initiating the call moves out of a picocell, then that call may be handed over to another CBS if the new location is within the hotspot region, or treated as a privileged overflow call to the EBS otherwise. This analysis leads to the following:

$$
\begin{gathered}
\lambda_{o}=\lambda_{n}^{\prime} p_{n}^{\prime r}+\lambda_{h}^{\prime} p_{h}^{\prime r} \\
\lambda_{p}=\lambda_{h}^{\prime} \frac{(1-\chi)}{\chi} \quad(\chi>0) \\
\lambda_{h}=h\left(\lambda_{n}\left(1-p_{n}^{r}\right)+\lambda_{h}\left(1-p_{h}^{r}\right)+\lambda_{t}\left(1-p_{t}^{r}\right)\right) \\
\lambda_{t}=v \lambda_{t} p_{t}^{r}+v \tau\left(1-p_{o}^{r}\right)\left(\lambda_{n} p_{n}^{r}+\lambda_{h} p_{h}^{r}\right) .
\end{gathered}
$$

\subsubsection{Picocell Model (CBS)}

Requests to overflow to a CBS come from mobile users that fail to connect to an EBS. We consider the collective rate of overflowing to all $K$ picocells. This rate is:

$$
\lambda_{o}^{\prime}=\tau\left(\lambda_{n} p_{n}^{r}+\lambda_{h} p_{h}^{r}\right),
$$

where $\tau$ is the fraction that attempt overflow (recall the discussion of partial overflow).

Handoff requests to a CBS include the following three cases: (a) new call requests to the CBS that are not blocked attempt to handoff when crossing the picocell boundary; (b) handoff calls to the CBS that are not dropped attempt to handoff when crossing the cell boundary; (c) successful takebacks (from the EBS) attempt to handoff when crossing the picocell boundary. Note that the partial handoff fraction is $\chi$. The handoff rate in steady-state is:

$$
\lambda_{h}^{\prime}=\chi h^{\prime}\left(\lambda_{n}^{\prime}\left(1-p_{n}^{\prime r}\right)+\lambda_{h}^{\prime}\left(1-p_{h}^{\prime r}\right)+\lambda_{t}^{\prime}\left(1-p_{t}^{\prime r}\right)\right) .
$$

Requests for call takeback from an EBS to a CBS include the following four cases: (a) new calls overflowed to EBS are taken back to CBS; (b) handoffs overflowed are taken back; (c) failed previous takeback attempts are tried once more; and (d) privileged overflows to EBS are taken back to CBS. Hence, the takeback rate is:

$$
\begin{aligned}
\lambda_{t}^{\prime}= & v^{\prime}\left(\lambda_{t}^{\prime} p_{t}^{\prime r}+\left(1-p_{p}^{\prime r}\right) \lambda_{p}\right. \\
& +\left(1-p_{o}^{\prime r}\right)\left(\lambda_{n}^{\prime} p_{n}^{\prime r}+\lambda_{h}^{\prime} p_{h}^{\prime r}\right)
\end{aligned}
$$

The total arrival rate to a CBS is the sum of the arrival rates of new calls, handoff calls, and takeback calls, as well as overflows and privileged overflows to the CBS.

\subsection{Equilibrium Loss Metrics}

Our analysis proceeds by considering our network model as a loss system with multiple servers (channels). We compute loss probabilities based on system load and network resources, similar to the well-known Erlang formula.
Let us first consider the process $N(t)$ in an EBS. Note that $\Lambda_{\varphi}$ is the system load generated from stream $\varphi$ : we have $\Lambda_{\varphi}=\lambda_{\varphi} \bar{T}_{\varphi}$. Letting $\pi\left(i, j, k, l, l_{p}\right)$ denote the joint stationary probability, we have:

$$
\pi\left(i, j, k, l, l_{p}\right)=(Q)^{-1} \frac{\left(\Lambda_{n}\right)^{i}\left(\Lambda_{h}\right)^{j}\left(\Lambda_{t}\right)^{k}\left(\Lambda_{o}\right)^{l}\left(\Lambda_{p}\right)^{l_{p}}}{i ! j ! k ! l !\left(l_{p}\right) !} .
$$

$Q$ is the normalization constant such that over state $s \in \Omega$, $\pi(s)$ sums to 1 , where the state space

$$
\begin{aligned}
\Omega= & \left\{\left(i, j, k, l, l_{p}\right): 0 \leq i, j, k, l, l_{p} \leq C_{m} ;\right. \\
& \left.i \leq C_{n}, l \leq C_{o}, l_{p} \leq C_{p}\right\} .
\end{aligned}
$$

Based on the equilibrium, we estimate system loads and performance metrics. For each stream $\varphi$, let $\Xi_{\varphi}$ denote the set of states in which calls of type $\varphi$ are rejected in an EBS. We calculate:

$$
\begin{aligned}
\text { load: } & \Lambda_{\varphi}=\lambda_{\varphi} \bar{T}_{\varphi} . \\
\text { rejection probability: } & p_{\varphi}^{r}=\sum_{s \in \Xi_{\varphi}} \pi(s) . \\
\text { carried traffic: } & (\mathrm{CT})_{\varphi}=\lambda_{\varphi}\left(1-p_{\varphi}^{r}\right) .
\end{aligned}
$$

The rejection sets $\Xi_{\varphi}$ in Equation 20 can be specified:

$$
\begin{aligned}
\Xi_{n}= & \left\{\left(i, j, k, l, l_{p}\right): i+j+k+l+l_{p}=C_{m}\right. \\
& \text { or } \left.i=C_{n}\right\} \\
\Xi_{o}= & \left\{\left(i, j, k, l, l_{p}\right): i+j+k+l+l_{p}=C_{m}\right. \\
& \text { or } \left.l=C_{o}\right\} \\
\Xi_{h}= & \left\{\left(i, j, k, l, l_{p}\right): i+j+k+l+l_{p}=C_{m}\right\} \\
\Xi_{t}= & \left\{\left(i, j, k, l, l_{p}\right): i+j+k+l+l_{p}=C_{m}\right\} \\
\Xi_{p}= & \left\{\left(i, j, k, l, l_{p}\right): i+j+k+l+l_{p}=C_{m}\right. \\
& \left.\quad \text { or } l_{p}=C_{p}\right\}
\end{aligned}
$$

Provided new call arrivals are Poisson, the probability of a new call being blocked equals the probability that the system is in a state that blocks new calls (i.e., the PASTA principle: Poisson arrivals see time average). For handoffs and redirected calls, a similar observation holds, as long as these streams are also Poisson. (We assume so.)

The analysis of traffic for a CBS is analogous. The CBS counterparts to Equations 19 to 21 are:

$$
\begin{aligned}
\Lambda_{\varphi}^{\prime} & =\bar{T}_{\varphi} \lambda_{\varphi}^{\prime} / K . \\
p_{\varphi}^{\prime r} & =\sum_{s^{\prime} \in \Xi_{\varphi}^{\prime}} \pi\left(s^{\prime}\right) . \\
(\mathrm{CT})_{\varphi}^{\prime} & =\lambda_{\varphi}^{\prime}\left(1-p_{\varphi}^{\prime r}\right) .
\end{aligned}
$$

where the equilibrium state distribution $\pi^{\prime}($.$) is similar to$ $\pi($.$) , but without privileged overflows to a CBS:$

$$
\pi^{\prime}(i, j, k, l)=\left(Q^{\prime}\right)^{-1} \frac{\left(\Lambda_{n}^{\prime}\right)^{i}\left(\Lambda_{h}^{\prime}\right)^{j}\left(\Lambda_{t}^{\prime}\right)^{k}\left(\Lambda_{o}^{\prime}\right)^{l}}{i ! j ! k ! l !} .
$$

Lastly, user-perceived loss metrics are calculated (for definition, refer to Section 3.5):

$$
\begin{aligned}
P_{\text {block }}^{u 1} & =p_{n}^{r} \cdot\left(1-\tau\left(1-p_{o}^{\prime r}\right)\right) . \\
P_{d r o p}^{u 1} & =p_{h}^{r} \cdot\left(1-\tau\left(1-p_{o}^{\prime r}\right)\right) .
\end{aligned}
$$

For Type-2 users, the scenario is a bit more complicated:

$$
P_{\text {block }}^{u 2}=\phi p_{n}^{\prime r} p_{o}^{r}+(1-\phi) p_{n}^{r}\left(1-\tau\left(1-p_{o}^{\prime r}\right)\right) .
$$


The term $\left(1-\tau\left(1-p_{o}^{r}\right)\right)$ arises because of the conditions for a call being rejected at an EBS, the probability that either the call does not attempt for an overflow to CBS, or the call attempts an overflow but fails. Explanation of other formulae are similar.

The user-perceived dropping probability measures the probability that an ongoing call is dropped, when a mobile user leaves a cell while continuing a call. For Type-2 users, we have the following three cases: (1) Type-2 user calls that are handed off between two CBS are rejected, and attempted overflows to EBS are also rejected; (2) Type-2 user calls that are handed off between two CBS are rejected, and attempted privileged overflows to EBS are also rejected; and (3) Type-2 user calls that are handed off between two EBS are rejected, and overflows to CBS are either not attempted or not successful. Capturing all these cases, we have:

$$
\begin{aligned}
P_{d r o p}^{u 2}= & \phi p_{h}^{\prime r}\left(p_{o}^{r}(1-\chi)+p_{p}^{r} \chi\right) \\
& +(1-\phi) p_{h}^{r}\left(1-\tau\left(1-p_{o}^{\prime r}\right)\right) .
\end{aligned}
$$

The foregoing analysis can be extended to general holding times without difficulty, because of the well-known insensitivity property. That is, the stationary state distribution depends only on the mean service time (channel holding time), and not on the service time distribution.

\subsection{Iterative Algorithm}

For this model, an iterative algorithm is developed for performance evaluation, based on the previous discussion:

\section{BEGIN}

Init for all $\varphi \in \mathcal{E}$

$$
p_{\varphi}^{r} \leftarrow 0, p_{\varphi}^{\prime r} \leftarrow 0
$$

$\epsilon \leftarrow$ specific error tolerance

REPEAT \{ Evaluate arrival rates, system loads and loss metrics for all streams $\}$

for all $\varphi \in \mathcal{E} \quad B E G I N$

$\left(p_{\varphi}^{r}\right)_{\text {sav }} \leftarrow p_{\varphi}^{r},\left(p_{\varphi}^{\prime r}\right)_{\text {sav }} \leftarrow\left(p_{\varphi}^{\prime r}\right)$

Evaluate $\lambda_{\varphi}, \lambda_{\varphi}^{\prime}$, (from Equations 11 to 17 )

Evaluate $\Lambda_{\varphi}, \Lambda_{\varphi}^{\prime}$, (from Equations 19 to 21 )

Update: $p_{\varphi}^{r}, p_{\varphi}^{\prime r}$, (from Equations 27 to 29 )

$$
\text { END UNTIL }
$$

$\left(\operatorname{diff}\left(\overrightarrow{p^{r}},\left(\overrightarrow{p^{r}}\right)_{\text {sav }}\right)<\epsilon \mathrm{AND} \operatorname{diff}\left(\overrightarrow{p^{\prime r}},\left(\overrightarrow{p^{\prime r}}\right)_{\text {sav }}\right)<\epsilon\right)$

End of the Algorithm;

where we define a function $\operatorname{diff}()$ for any two vectors $\vec{p}=$ $\left(p_{\varphi}\right), \vec{q}=\left(q_{\varphi}\right), \varphi \in \mathcal{E}$, as follows:

$\operatorname{diff}(\vec{p}, \vec{q})=\sum_{\varphi \in \mathcal{E}}\left|p_{\varphi}-q_{\varphi}\right|$.

\section{NUMERICAL RESULTS}

We investigate by numerical experiments the sensitivity of the loss metrics to the model input parameters. We choose a default set of parameter values, and then selectively vary some of them.

The default settings are as follows. For the EBS, we set $C_{m}=12, C_{n}=9$, and $C_{o}=5$. For each CBS, we set $C_{m}^{\prime}=4, C_{n}^{\prime}=3$, and $C_{o}^{\prime}=2$. The number of picocells per macrocell is $K=3$. In this case, the total number of channels is $C_{m}+K C_{m}^{\prime}=24$. The mean call duration is $\bar{T}^{0}=14.0$ for all calls. The mean cell residence time at the macrocell is $\bar{R}=35.0$, and $A=0.25$. The mean cell residence time $\bar{R}^{\prime}$ in a picocell is estimated from Equation 4 where we use the settings $\beta=0.75$ and $\alpha=0.7$. We set parameters $\tau=0.3$ and $\chi=0.8$, which satisfy Equation 5 and Equation 6, respectively.
The rates of call arrivals are designated as follows. The call arrival rate for Type- 1 users is $\lambda^{u 1}=0.3$. The call arrival rate for Type- 2 users is $\lambda^{u 2}=1.0$. The proportion of Type- 2 calls initiated from within the hotspot region is $\phi=0.85$.

If we temporally ignore loss effects, then the raw system load imposed by Type- 1 and Type- 2 users is $\left(\lambda^{u 1}+\lambda^{u 2}\right) \bar{T}^{0}=$ $1.3 \times 14=18.2$ Erlangs. Comparing this with the total number of channels (24), we note that the default case has a medium load factor (75\%). As $\lambda^{u 2}$ increases (i.e., traffic in the hotspot area is more intense), we expect the load factor to increase as well. For example, if $\lambda^{u 2}=1.3$, we can analogously calculate that Type- 2 mobile users themselves impose a load of 18.2 Erlangs, which brings the total load to 22.4 Erlangs. In this situation, the system load is extremely high (93\%).

\subsection{Effect of Traffic Load}

The first experiment is designed to study the issue of how loss metrics respond to the traffic intensity, especially when traffic from Type- 2 users becomes intense. We fix $\lambda^{u 1}$ to be 0.3 but vary $\lambda^{u 2}$ from 0.5 to 1.5 . We also suppose that most Type-2 users initiate calls via a CBS $(\phi=0.85)$. The reported results include the rejection metrics observed at EBS and CBS, and user-perceived loss for both Type-1 and Type-2 users. These appear in Figure 4.

The general trends in Figure 4 are as expected. Loss rates increase monotonically with system load, and the loss rates in the busy hotspot region are usually higher than those at the EBS. In general, the call blocking rates tend to be higher than the call dropping rates.

From the numerical results, we note that the user-perceived loss probabilities in Figure 4(b) are lower than the rejection metrics observed at an EBS or a CBS in Figure 4(a). The performance improvement (i.e., reduction of user-perceived loss) is evident, even though the CBS coverage is only partial. This is due to the overflow mechanism, which provides calls rejected at one BS with a second chance for connection to another BS, as well as the takeback mechanism, which prevents excessive exploitation of channels in a BS. For Type-2 users, the dropping probability is actually higher than the blocking probability. However, both loss rates are still lower than those experienced by Type-1 users.

\subsection{Effect of Number of Picocells}

The second experiment is designed to study the effect of using more picocells to cover the hotspot region, which has a fixed area (i.e., $K \epsilon \alpha$ is a constant, and the coverage efficiency is also fixed at $\alpha=0.7$ ). We vary $K$ to examine how the loss metrics respond. The loss metrics are the same as those in the first experiment. The results are reported in Figure 5.

The general trends in Figure 5 show that loss rates decrease (as expected) when additional picocells are deployed. This trend makes sense, since more channel capacity is available in the network. However, the decreases show a diminishing returns effect, and the effects differ at the EBS and the CBS, as well as between blocking and dropping.

From the numerical results, as $K$ increases, the rejection probabilities (both blocking and dropping) decrease significantly at the CBS level, but only slightly at the EBS. The user-perceived loss rates in Figure 5(b) follow the same trend as the system loss rates in Figure 5(a). Type-2 users perceive consistently lower rejection than those of Type-1. However, 


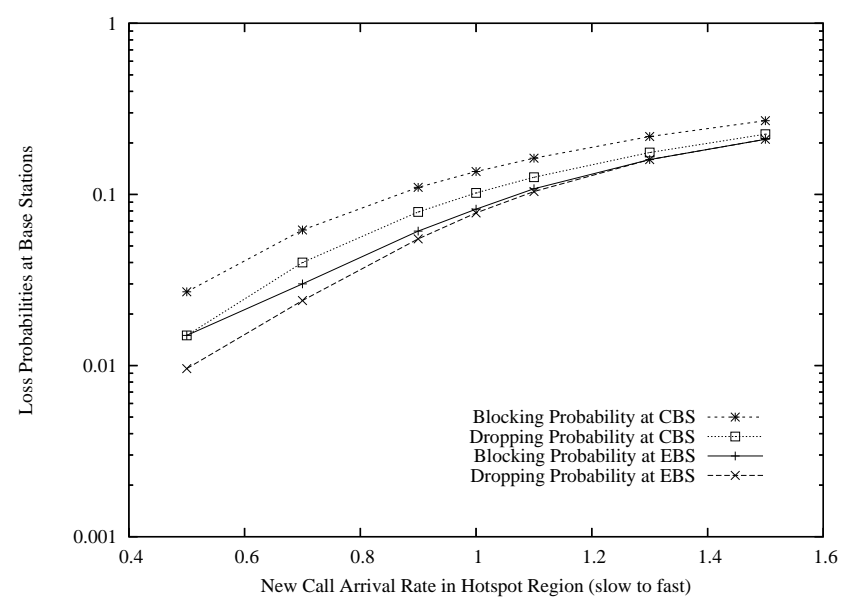

(a) System Performance

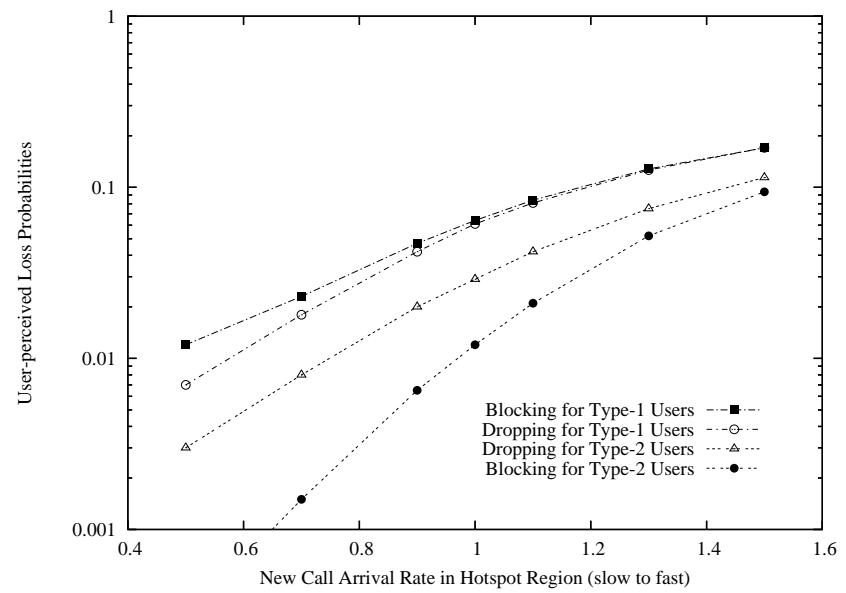

(b) User-Perceived Performance

Figure 4: Effect of Traffic Intensity

when $K$ exceeds a certain value (say, $K=5$ in this example), the primary effect is in the reduction of blocking probability for Type-2 mobile users (which is already low); the decrease in the dropping probability of calls from Type- 2 mobile users becomes less significant. We would not recommend $K$ larger than 5 , since the deployment costs increase linearly with $K$, while adding more CBS beyond $K=5$ does not lead to any significant improvement in system performance.

\subsection{Discussion}

There are several performance trends to be noted. First, as $K$ increases, a crossover occurs in Figure 5 between the rejection performance curves of Type- 1 users and those of Type- 2 users. When $K$ is small, Type- 2 users experience higher rejection than the other class, since their calls are directed to a (busy) CBS first, while Type- 1 user calls are directed to the EBS first. When $K$ is larger, Type- 2 mobile users experience less blocking. However, the decrease in dropping is not as significant as the reduction of blocking. (Due to partial CBS coverage, some handoffs between CBS are redirected to EBS as prioritized handoffs; their rejection probability is not significantly improved by increasing $K$.)

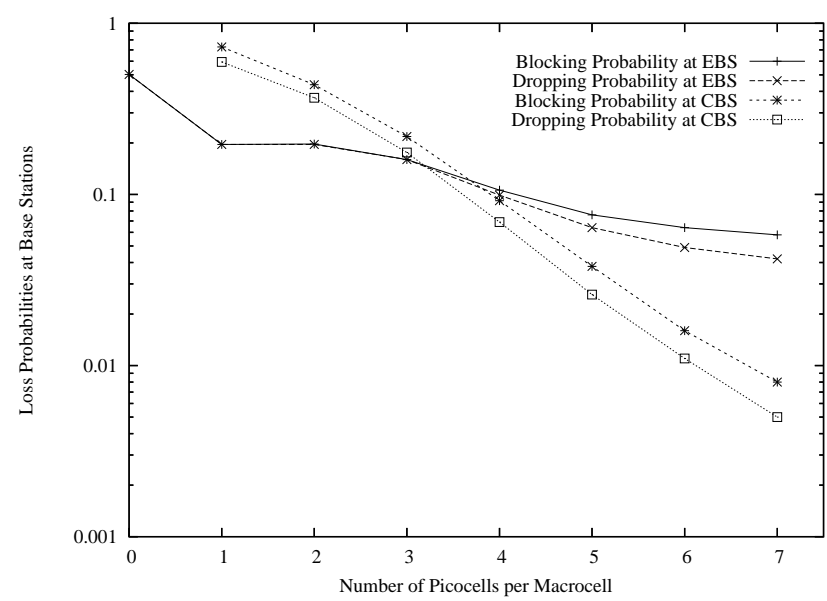

(a) System Performance

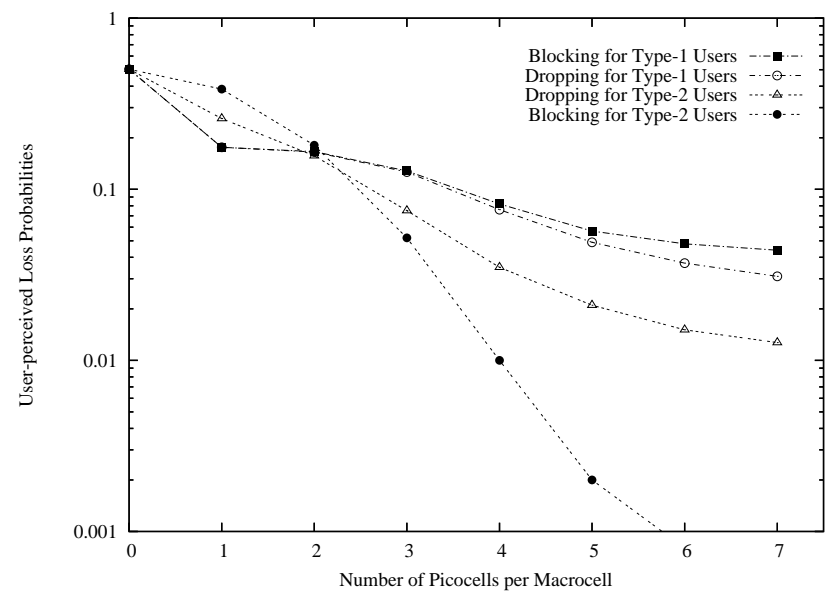

(b) User-Perceived Performance

Figure 5: Effect of Number of Picocells

Second, the user-perceived rejection rate of Type- 2 users is consistently lower than that of Type-1 users for the parameter range in our experiment (see Figure 4 and Figure 5). However, the difference becomes smaller as the traffic becomes more intense (Figure 4), especially when the new call arrival rate of Type- 2 users increases. Third, we note that both types of mobile users experience lower loss when increasing the number of CBS per macrocell, but Type- 2 mobile users benefit the most.

\section{SUMMARY AND CONCLUSIONS}

In this paper, we have studied the system performance of a cellular network with partial coverage picocells under different traffic conditions. The model in this paper provides a useful tool for performance study of cellular networks and the placement of CBS. We model the heterogeneity of traffic within a macrocell. Using the proposed traffic management scheme with CBS, the wireless/cellular system achieves consistent loss reduction and improved system performance under medium to high load.

We have developed an analytical model for this scheme, and studied several interesting related issues. In particular, 
we apply our model to the case of fairly intense traffic, concentrated in a relatively small hotspot area. Under the situation considered, we found by numerical results that the proposed traffic management scheme reduces loss and enhances system performance. This observation agrees with other traffic management schemes with picocells. To the best of our knowledge, this work is the first to develop a model under both CBS partial coverage and using bi-directional overflows and takebacks as the traffic management scheme.

Our model for the proposed traffic management approach has relatively low complexity, which makes it possible to evaluate system performance for a large set of different parameter combinations. This low-complexity algorithm can also become a building block for developing, solving, and evaluating more complicated models, such as a model for multiple EBS, or different call classes.

From our analysis and discussion, we observe the diminishing returns effect, and the rising cost for deployment of more CBS. Ultimately, we expect to use this model to estimate the number of CBS needed to optimize system performance under a fixed total budget, or to maximize the cost-adjusted returns.

There are many promising directions for future work, which will address some of the limitations induced by the simplifying assumptions in our model. Some of these extensions include non-Poisson call arrivals, general distributions for call duration and cell residence times, overlapping EBS configurations, and the relationships between cellular coverage and user mobility pattern [13]. We have not yet studied the system cost model in any detail; this also needs attention.

\section{Acknowledgements}

The authors are grateful to the MobiWac 2008 reviewers for their constructive comments on an earlier version of this paper. Financial support for this research was provided by NSERC (Natural Sciences and Engineering Research Council), iCORE (Informatics Circle of Research Excellence), and TELUS Mobility.

\section{REFERENCES}

[1] X. Chao, M. Miyazawa, and M. Pinedo, Queueing Networks: Customer Signals and Product Form Solution. Wiley, 1999.

[2] S. Chung and J. Lee, "Performance Analysis and Overflowed Traffic Characterization in Multiservice Hierarchical Wireless Systems", IEEE Transactions on Wireless Communications, Vol. 4, pp. 904-918, 2006.

[3] A. Erlang, "Solution of Some Problems in the Theory of Probabilities of Significance in Automatic Telephone Exchanges", In The Life and Works of A.K. Erlang, E. Brockmeyer, H. Halstrom, and A. Jenson, editors, 1948.

[4] Y. Fang and Y. Zhang, "Call Admission Control Schemas and Performance Analysis in Wireless Mobile Networks", IEEE Transactions on Vehicular Technology, Vol. 51, pp. 371-382, 2002.
[5] L. Greenstein, C. Lin, and R. Gitlin, "A Microcell/Macrocell Cellular Architecture for Low and High-mobility Wireless Users", IEEE Journal on Selected Areas in Communication, Vol. 11, pp. 885-891, 1993.

[6] E. Halepovic and C. Williamson, "Characterizing and Modeling User Mobility in a Cellular Data Network", Proceedings of ACM PE-WASUN 2005, Montreal, QC, pp. 71-78, October 2005.

[7] D. Hong and S. Rappaport, "Traffic Model and Performance Analysis for Cellular Mobile Telephone Systems with Prioritized and Non-prioritized Handoff Procedures", IEEE Transactions on Vehicular Technology, Vol. 35, pp. 77-92, 1986.

[8] X. Lagrange and P. Godlewski, "Performance of a Hierarchical Cellular Network with Mobility-dependent Handover Strategies", Proceedings of Vehicular Technology Conference, Vol. 3, pp. 1868-1872, 1996.

[9] W. Li and X. Chao, "Modeling and Performance Evaluation of a Cellular Mobile Network", IEEE/ACM Transactions on Networking, Vol. 12, pp. 131-145, 2004.

[10] J. Luo and A. Alfa, "Performance Analysis of a Mobile Cell Station in a Wireless Network with Realistic Handoff Calls", Proceedings of SCS SPECTS 2005, Cherry Hill, NJ, pp. 235-244, July 2005.

[11] T. Rappaport, Wireless Communications: Principles and Practice, 2nd edition, Prentice Hall, 2001.

[12] W. Shan, P. Fan, and Y. Pan. "Performance Evaluation of a Hierarchical Cellular Communication Networks", IEEE Transactions on Parallel and Distributed Systems, Vol. 14, pp. 72-83, 2003.

[13] S. Thajchyapong and J. Peha, "Mobility Patterns in Microcellular Wireless Networks", IEEE Transactions on Mobile Computing, Vol. 5, pp. 52-63, 2006.

[14] V. Vakili, A. Aziminejad, and M. Dehbozorgi, "A Novel Speed-sensitive Bidirectional Overflow and Handdown Resource Allocation Strategy for Hierarchical Cellular Networks", Proceedings of 1st International Symposium on Information and Communication Technology, Dublin, Ireland, pp. 126-131, 2003.

[15] S. Wie, J. Jang, B. Shin, and D. Cho, "Handoff Analysis of the Hierarchical Cellular System", IEEE Transactions on Vehicular Technology, Vol. 49, pp. 2027-2036, 2000.

[16] C. Williamson, E. Halepovic, H. Sun, and Y. Wu, "Characterization of CDMA2000 Cellular Data Network Traffic", Proceedings of 5th International IEEE Workshop on Wireless Local Networks (WLN 2005), Sydney, Australia, November 2005.

[17] K. Yeo and C. Jun, "Modeling and Analysis of Hierarchical Cellular Networks with General Distributions of Call and Cell Residence Times", IEEE Transactions on Vehicular Technology, Vol. 51, pp. 1361-1374, 2002. 